\title{
Iodine-125 interstitial brachytherapy reduces tumor growth via Warburg effect inhibition in non-small cell lung cancer A549 xenografts
}

\author{
JUN ZHANG $^{1 *}$, YANGJUN ZHU ${ }^{2 *}$, MENGJIE DONG ${ }^{1}$, JUN YANG $^{1}$, WANWEN WENG ${ }^{1}$ and LISONG TENG ${ }^{3,4}$ \\ Departments of ${ }^{1}$ Nuclear Medicine, ${ }^{2}$ Ultrasonography and ${ }^{3}$ Surgical Oncology; \\ ${ }^{4}$ Key Laboratory of Precision Diagnosis and Treatment for Hepatobiliary and Pancreatic Tumor of Zhejiang Province, \\ The First Affiliated Hospital, College of Medicine, Zhejiang University, Hangzhou, Zhejiang 310003, P.R. China
}

Received March 26, 2018; Accepted August 10, 2018

DOI: $10.3892 / \mathrm{ol} .2018 .9346$

\begin{abstract}
Iodine-125 interstitial brachytherapy ( ${ }^{125} \mathrm{I}$-IBT) is an alternative and effective treatment option for unresectable non-small cell lung cancer (NSCLC), and the Warburg effect is a determinant of tumor growth. The present study aimed to explore the influence of ${ }^{125} \mathrm{I}$-IBT on tumor growth and the Warburg effect, and the potential mechanisms underlying NSCLC progression. Mice with A549 cell xenografts were evenly divided into a control group without ${ }^{125} \mathrm{I}$-IBT, and three treatment groups receiving ${ }^{125} \mathrm{I}-\mathrm{IBT}$ with 20,40 and $60 \mathrm{~Gy}$. Tumor volume (TV), maximum standardized uptake value (SUVmax) determined by ${ }^{18} \mathrm{~F}$-fluorodeoxyglucose $\left({ }^{18} \mathrm{~F}-\mathrm{FDG}\right)$ micro-positron emission tomography/computed tomography and mean optical density (MOD) of mammalian target of
\end{abstract}

Correspondence to: Professor Lisong Teng, Department of Surgical Oncology, The First Affiliated Hospital, College of Medicine, Zhejiang University, 79 Qingchun Road, Hangzhou, Zhejiang 310003, P.R. China

E-mail: 1steng@zju.edu.cn

*Contributed equally

Abbreviations: NSCLC, non-small cell lung cancer; ${ }^{125} \mathrm{I}-\mathrm{IBT}$, iodine-125 interstitial brachytherapy; OXPHOS, oxidative phosphorylation; PET/CT, positron emission tomography/computed tomography; ${ }^{18} \mathrm{~F}-\mathrm{FDG},{ }^{18} \mathrm{~F}$-fluorodeoxyglucose; PDK1, pyruvate dehydrogenase kinase 1; TV, tumor volume; TIR, tumor inhibition rate; SUVmax, maximum standardized uptake value; FUAR, ${ }^{18} \mathrm{~F}-\mathrm{FDG}$ uptake attenuation rate; IHC, immunohistochemistry; mTOR, mammalian target of rapamycin; HIF-1 $\alpha$, hypoxia inducible factor-1 $\alpha$; GLUT1, glucose transporter 1; MOD, mean optical density; ESR, expression suppression rate; PI3K, phosphoinositide 3-kinase; AKT, protein kinase B; MAPKK, mitogen-activated protein kinase kinase; ERK, extracellular regulated protein kinase; PKM2, pyruvate kinase M2; HK2, hexokinase 2; ROS, reactive oxygen species; G6Pase, glucose-6-phosphatase

Key words: iodine-125 seed, brachytherapy, Warburg effect, ${ }^{18} \mathrm{~F}$-fluorodeoxyglucose, micro-PET/CT, non-small cell lung cancer rapamycin (mTOR), c-Myc, hypoxia inducible factor- $1 \alpha$ (HIF-1 $\alpha)$ and glucose transporter 1 (GLUT1) staining were compared among groups. Tumor inhibition rate (TIR), ${ }^{18} \mathrm{~F}-\mathrm{FDG}$ uptake attenuation rate (FUAR) and expression suppression rate (ESR) were also calculated on day 14 and 28 . The results demonstrated that the mean TV in the 60 and 40 Gy groups was smaller compared with the control TVs since days 14 and 16, respectively. The mean SUVmax value of the 60 Gy group at day 14, and all treatment group SUVmax values at day 28 were lower compared with the controls. In addition, the MOD of mTOR and GLUT1 was lower in the 60 Gy group, compared with the other groups, and c-Myc and HIF-1 $\alpha$ values were lower in the 40 and 60 Gy groups, compared with the control and 20 Gy group $(\mathrm{P}<0.05)$. SUVmax positively correlated to TV (day 14, r=0.711; day 28, r=0.586) and the MOD of c-Myc and GLUT1 ( $r=0.621$ and 0.546, respectively; $\mathrm{P}<0.01$ ). Furthermore, dose dependent increases were observed for TIR, FUAR and ESR. In conclusion, ${ }^{125}$ I-IBT reduced tumor growth by inhibiting the Warburg effect, which may have resulted from downregulation of mTOR, c-Myc, HIF-1 $\alpha$ and GLUT1 expression, particularly c-Myc and GLUT1, in NSCLC A549 xenografts.

\section{Introduction}

Lung cancer is the most frequently diagnosed cancer and the leading cause of cancer mortality worldwide. Over $80 \%$ of cases are histologically classified as non-small cell lung cancer (NSCLC) $(1,2)$. Surgery is generally the treatment of choice for early stage NSCLC. However, 30-55\% of patients develop recurrence even following complete resection, and the mortality rate is high (3). As early disease is typically asymptomatic, the majority of patients are diagnosed at a distant stage, resulting in extremely poor prognosis, even with chemotherapy and/or radiotherapy (2). Recently, computed tomography (CT)-guided iodine-125 interstitial brachytherapy ( ${ }^{125}$ I-IBT) has been employed to treat NSCLC at the inoperable advanced stage, and in cases with local recurrence or metastasis following surgery or concurrent chemoradiotherapy, alone or in combination with other treatments (3-6). It was demonstrated that ${ }^{125}$ I-IBT significantly improves overall 
survival and local control, by maximizing the radiation dose delivered to the tumor, and minimizing radiation injury of the surrounding normal lung tissue. Certain preclinical studies have further suggested that increased $\mathrm{G} 2 / \mathrm{M}$ arrest, increased apoptosis and enhanced bystander effect may serve key roles in the anti-proliferative effects induced by ${ }^{125} \mathrm{I}-\mathrm{IBT}$ in NSCLC (7-9). However, the association between ${ }^{125}$ I-IBT and glucose metabolism in NSCLC remains unclear.

The Warburg effect, a hallmark for tumor cells, was discovered by Otto Warburg in 1924. It is well established that in contrast to normal cells, which predominantly rely on mitochondrial oxidative phosphorylation (OXPHOS) to generate the energy required for cellular processes, the majority of tumor cells instead rely on aerobic glycolysis. The Warburg effect is a determinant of tumor cell proliferation (10). The rate of glucose entry into tumor cells is at least 20-30-fold higher than normal cells. This unique phenomenon is the basis for the use of positron emission tomography (PET)/CT with ${ }^{18} \mathrm{~F}$-fluorodeoxyglucose $\left({ }^{18} \mathrm{~F}\right.$-FDG), a radioactive glucose analog that is intensely accumulated by tumor cells (11). ${ }^{18} \mathrm{~F}-\mathrm{FDG} \mathrm{PET} / \mathrm{CT}$ is widely used for initial diagnosis, disease staging, recurrence and metastasis detection, as well as for monitoring chemotherapy and/or radiotherapy response in lung cancer (12-16).

In recent years, several studies have investigated the Warburg effect in lung cancer progression, as well as for the development of targeted therapies (17-19). It was suggested that Sad1 and UNC84 domain containing 2 (SUN2) protein may suppress lung cancer cell proliferation and migration by attenuating the Warburg effect, whereas pyruvate dehydrogenase kinase 1 (PDK1), may promote lung cancer cell proliferation and migration, via the inverse mechanism. In addition, dichloroacetate, a metabolic agent, may partially revert the Warburg effect in tumor cells, increase X-ray sensitivity and inhibit the growth of lung cancer cells. Based on these previous reports, the present study aimed to investigate if and how the Warburg effect was involved in ${ }^{125}$ I-IBT for NSCLC. Herein, it was hypothesized that ${ }^{125} \mathrm{I}$-IBT may have suppressed tumor growth via Warburg effect inhibition in NSCLC. Alterations in tumor growth and ${ }^{18} \mathrm{~F}$-FDG uptake were monitored using micro-PET/CT imaging. In addition, the relationships among these factors, the expression of glycolysis-associated molecules and the radiation dose were analyzed in NSCLC A549 xenografts following ${ }^{125} \mathrm{I}$-IBT.

\section{Materials and methods}

Cell culture and animal model. The A549 cell line was purchased from Shanghai Jianglin Biological Technology Co., Ltd. (Shanghai, China). Cells were cultured in RPMI-1640 (GE Healthcare Life Sciences, Logan, UT, USA) supplemented with $10 \%$ fetal bovine serum (FBS; Gibco; Thermo Fisher Scientific, Inc., Waltham, MA, USA), $100 \mathrm{U} / \mathrm{ml}$ penicillin and $0.1 \mathrm{mg} / \mathrm{ml}$ streptomycin at $37^{\circ} \mathrm{C}$ in a $5 \% \mathrm{CO}_{2}$ atmosphere. Cells were trypsinized and harvested once $80-90 \%$ confluence was reached. Male BALB/c-nu mice (18-20 g; 4-6 weeks old) were purchased from Shanghai SLAC Laboratory Animal Co., Ltd. (Shanghai, China) and allowed to acclimatize for 1 week in a specific pathogen-free room under controlled temperature and humidity prior to experimental initiation. Following this, $\sim 0.2 \mathrm{ml}$ A549 cell suspension ( $1 \times 10^{7}$ cells/1 ml RPMI-1640) was injected subcutaneously into the right armpits of the forelegs. After 4 weeks, mice with a xenograft diameter of $\sim 10 \mathrm{~mm}$ were used for study.

Study design. A total of 24 prior mice were randomly divided into four groups ( $n=6$ per group). The control group without ${ }^{125}$ I seed implantation, and three treatment groups with a prescribed dose of 20, 40 and $60 \mathrm{~Gy}$, via 1-2 ${ }^{125} \mathrm{I}$ seed implantation with a radioactivity of $0.3-0.6 \mathrm{mCi}$, respectively. The treatment period was 4 weeks. Tumor growth was monitored by determining the xenograft size every 2 days with a calliper. Before and 14, 28 days after receiving ${ }^{125} \mathrm{I}-\mathrm{IBT}$, micro-PET/CT imaging was performed to determine the baseline level and post-treatment alterations in ${ }^{18} \mathrm{~F}-\mathrm{FDG}$ uptake. At the end of study, the mice were euthanized with dislocation of cervical vertebra after being anesthetized with $3 \%$ isoflurane inhalation and all xenografts were histologically analyzed with immunohistochemical (IHC) staining.

Interstitial brachytherapy. ${ }^{125}$ I seeds (model 6711; diameter, $0.8 \mathrm{~mm}$; length, $4.5 \mathrm{~mm}$; half-life, 59.6 days; half value thickness, $1.7 \mathrm{~cm}$ in tissue; main emission, 27.4-31.4 Kev X-ray and $35.5 \mathrm{Kev} \gamma$-ray) were provided by Seeds Biological Pharmacy, Ltd. (Tianjin, China). The ${ }^{125} \mathrm{I}$ seeds were preloaded into 18-gauge needles and subsequently implanted into the xenograft center. The radiation prescribed dose was planned based on the axial micro-CT images using a brachytherapy treatment planning system (Beijing Astro Technology, Beijing, China).

Tumor volume. Based on measurements of the short (a) and long (b) tumor diameters, tumor volume (TV) was calculated, according to the formula of $T V=1 / 2 \mathrm{x} \mathrm{a}^{2} \mathrm{x} \mathrm{b}$. The TV and tumor inhibition rate (TIR) on day 14 and 28 were defined as $\mathrm{TV}_{14}$, $\mathrm{TV}_{28}, \mathrm{TIR}_{14}$ and $\mathrm{TIR}_{28}$, respectively. TIR was calculated using the following equation: $\mathrm{TIR}=\left(\right.$ mean of $\mathrm{TV}_{\mathrm{c}}$-mean of $\left.\mathrm{TV}\right) /$ mean of $\mathrm{TV}_{\mathrm{c}} \times 100 \%$, where $\mathrm{TV}_{\mathrm{c}}$ was the control $\mathrm{TV}$, and $\mathrm{TV}_{\mathrm{t}}$ was the treatment group TV.

Micro-PET/CT imaging. ${ }^{18} \mathrm{~F}-\mathrm{FDG}$ was synthesized by the $\mathrm{PET} / \mathrm{CT}$ center in our institution. Following an overnight fast, mice were injected with $\sim 0.1 \mathrm{mCi}{ }^{18} \mathrm{~F}-\mathrm{FDG}$ via the tail vein. After $1 \mathrm{~h}$, mice were anesthetized with $3 \%$ isoflurane inhalation and placed in the prone position in the center of a Siemens Inveon combined micro-PET/CT scanner (Siemens Preclinical Solution USA, Inc., Knoxville, TN, USA). Micro-CT scans were performed with a $80 \mathrm{kV}$ X-ray tube voltage, $500 \mu \mathrm{A}$ current, $150 \mathrm{~ms}$ ec exposure time and 120 rotation steps. Micro-PET static acquisition was subsequently performed for $10 \mathrm{~min}$ and the data were processed using the ordered set expectation maximization algorithm for three dimensional PET reconstruction. Micro-PET/CT images were analyzed with an Inveon Research Workplace 4.1 (Siemens, Erlangen, Germany). The maximum standardized uptake value (SUVmax; $\mathrm{g} / \mathrm{ml}$ ) of the xenograft was measured on day 0, 14 and 28 and referred to as SUVmax 0 , SUVmax SUVmax ${ }_{28}$, respectively. The ${ }^{18}$ F-FDG uptake attenuation rate (FUAR) of the xenograft on day 14 and 28 was defined as $\mathrm{FUAR}_{14}$ and $\mathrm{FUAR}_{28}$, respectively, using the following equation: $F U A R=\left(\right.$ mean $\mathrm{SUVmax}_{\mathrm{c}}$ value-mean $\mathrm{SUVmax}_{\mathrm{t}}$ 


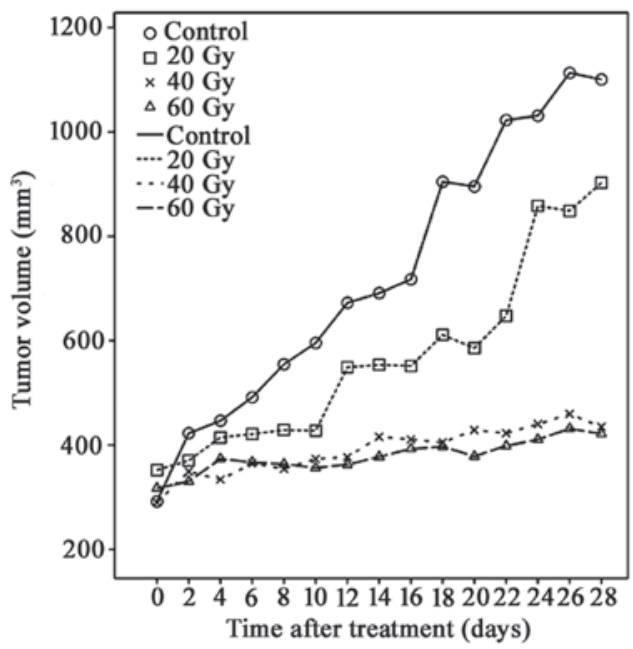

Figure 1. Tumor growth curve of A549 xenografts following the administration of ${ }^{125}$ I-IBT. Tumor volume gradually enlarged over time in all groups, although growth was reduced as the radiation dose increased. ${ }^{125}$ I-IBT, iodine-125 interstitial brachytherapy.

value)/mean SUVmax value $100 \%$, where $\mathrm{SUVmax}_{\mathrm{c}}$ was the control group SUVmax, and the SUVmax ${ }_{t}$ was the treatment group SUVmax.

Histological evaluation. All xenografts were fixed in $10 \%$ formalin overnight at $4^{\circ} \mathrm{C}$ and subsequently embedded in paraffin for histological section preparation $(4 \mu \mathrm{m})$. The sections were deparaffinized and stained with hematoxylin and eosin for normal histological evaluation. IHC was also performed with antibodies to detect mammalian target of rapamycin (mTOR), c-Myc, hypoxia inducible factor-1 $\alpha$ (HIF-1 $\alpha$ ) and glucose transporter 1 (GLUT1) expression, according to manufacturer's instructions. Primary antibodies (Abcam, Cambridge, UK) used were anti-mTOR ab2732 (diluted 1:200), anti-c-Myc ab32072 (diluted 1:100), anti-HIF-1 $\alpha$ ab16066 (diluted 1:400) and anti-GLUT1 ab652 (diluted 1:200). In brief, sections were dewaxed and rehydrated prior to quenching in $3 \%$ hydrogen peroxide to block endogenous peroxidase activity for $10 \mathrm{~min}$. Antigen retrieval was performed by immersing the sections in EDTA/Tris solution ( $\mathrm{pH} 9.0)$ in a microwave oven for $2 \times 15 \mathrm{~min}$. Sections were cooled at room temperature and washed in PBS for $3 \times 10$ min. Following this, sections were blocked in 5\% bovine serum albumin (BSA) for $30 \mathrm{~min}$ and subsequently incubated overnight with primary antibodies at $4{ }^{\circ} \mathrm{C}$ and rewarmed for $30 \mathrm{~min}$ and washed in PBS for $3 \times 10 \mathrm{~min}$. Then sections were incubated with biotinylated secondary antibodies at $37^{\circ} \mathrm{C}$ for $45 \mathrm{~min}$ and washed in PBS for $3 \times 10 \mathrm{~min}$, followed by exposure to streptavidin-biotin complex (Ready-to-use SABC-POD kits: SA1021, goat anti-mouse IgG and SA1022, goat anti-rabbit $\mathrm{IgG}$ ) for $45 \mathrm{~min}$ at $37^{\circ} \mathrm{C}$ and diaminobenzidine tetrahydrochloride (DAB AR1022; both Boster, Wuhan, China). Staining without primary antibodies was performed in parallel and served as a negative control. Finally, sections were counterstained with hematoxylin, dehydrated in ethanol and xylene and mounted with cover slips using neutral balsam. Each section was assessed in three random microscopic fields (x200). The expression of mTOR, c-Myc, HIF-1 $\alpha$ and GLUT1 was quantified by determining the mean optical density (MOD) using Image-Pro Plus software (version 6.0; Media Cybernetics, Inc. Maryland, USA). The expression suppression rate (ESR) of mTOR, c-Myc, HIF-1 $\alpha$ and GLUT1 was defined as $\mathrm{ESR}_{\mathrm{mTOR}}, \mathrm{ESR}_{\mathrm{c}-\mathrm{Myc}}, \mathrm{ESR}_{\mathrm{HIF}-1 \alpha}$ and $\mathrm{ESR}_{\mathrm{GLUT1}}$, respectively, using the following equations: $\mathrm{ESR}=($ mean $\mathrm{MOD}_{\mathrm{c}}$-mean $\mathrm{MOD}_{\mathrm{t}}$ /mean $\mathrm{MOD}_{\mathrm{c}} \mathrm{x} 100 \%$, where $\mathrm{MOD}_{\mathrm{c}}$ was the control group MOD, and $\mathrm{MOD}_{\mathrm{t}}$ was the MOD of the treatment groups.

Statistical analysis. All statistical analyses were performed using SPSS 19.0 software (IBM Corp., Armonk, NY, USA). The continuous variables were described as the mean \pm standard deviation. Significant differences were evaluated using one-way analysis of variance. If variances were equal, all pairwise comparisons between group means were performed with Fisher's Least Significant Difference test; if unequal, Dunnett's T3 test was used. Pearson's correlation analysis was used to evaluate the relationships among TV, SUVmax and the MOD of mTOR, c-Myc, HIF- $1 \alpha$ and GLUT1. P<0.05 was considered to indicate a statistically significant difference.

\section{Results}

${ }^{125}$ I-IBT inhibits A549 xenograft growth. A549 xenograft growth over time following ${ }^{125}$ I-IBT was presented in Fig. 1. Overall, the tumor gradually enlarged over time in all groups, although TV decreased as the radiation dose increased. The mean TV of the $60 \mathrm{~Gy}$ group $\left(377.3 \pm 227.2 \mathrm{~mm}^{3}\right)$ and $40 \mathrm{~Gy}$ group $\left(410.7 \pm 174.42 \mathrm{~mm}^{3}\right)$ was significantly reduced compared with controls since day $14\left(691.0 \pm 258.5 \mathrm{~mm}^{3}\right)$ and 16 $\left(717.5 \pm 212.9 \mathrm{~mm}^{3}\right)$, respectively $(\mathrm{P}<0.05)$. However, no statistical differences were observed between the 20 Gy group and controls or among all treatment groups $(\mathrm{P}>0.05)$. In addition, the $\mathrm{TIR}_{14}$ of the 20,40 and $60 \mathrm{~Gy}$ groups was $19.9,39.8$ and $45.4 \%$; and the $\mathrm{TIR}_{28}$ were $18.0,60.5$ and $61.7 \%$, respectively. These data suggested that ${ }^{125}$ I-IBT may have inhibited the growth of A549 xenografts, and inhibition was enhanced as the radiation dose increased.

${ }^{125} \mathrm{I}-\mathrm{IBT}$ reduces ${ }^{18} \mathrm{~F}-\mathrm{FDG}$ uptake in A549 xenografts. ${ }^{18} \mathrm{~F}-\mathrm{FDG}$ uptake within A549 xenografts following ${ }^{125}$ I-IBT was presented in Fig. 2. In general, compared with controls, the ${ }^{18} \mathrm{~F}-\mathrm{FDG}$ uptake of xenografts gradually reduced over time in ${ }^{125} \mathrm{I}$-IBT treatment groups. Furthermore, the reduction in SUVmax was increased as the radiation dose increased (Fig. 3). The mean of SUVmax ${ }_{14}$ of the 60 Gy-group was significantly lower than the control group $(0.91 \pm 0.13 \mathrm{~g} / \mathrm{ml}$ vs. $1.08 \pm 0.08 \mathrm{~g} / \mathrm{ml} ; \mathrm{P}<0.05)$. The mean SUVmax ${ }_{28}$ of all treatment groups was significantly lower than the control group $(20 \mathrm{~Gy}, 0.94 \pm 0.19 \mathrm{~g} / \mathrm{ml} ; 40 \mathrm{~Gy}$, $0.93 \pm 0.08 \mathrm{~g} / \mathrm{ml} ; 60 \mathrm{~Gy}, 0.79 \pm 0.16 \mathrm{~g} / \mathrm{ml}$ vs. control group, $1.15 \pm 0.22 \mathrm{~g} / \mathrm{ml} ; \mathrm{P}<0.05)$, although there were no significant differences among treatment groups on day 14 and 28 ( $\mathrm{P}>0.05)$. Furthermore, the $\mathrm{FUAR}_{14}$ of the 20, 40 and 60 Gy groups was $6.5,8.3$ and $15.7 \%$, and the $\mathrm{FUAR}_{28}$ of the 20,40 and $60 \mathrm{~Gy}$ groups was $18.3,19.1$ and $31.3 \%$, respectively. These data suggested that ${ }^{125} \mathrm{I}$-IBT may have reduced ${ }^{18} \mathrm{~F}$-FDG uptake within A549 xenografts, and this effect was more marked as the radiation dose increased. 

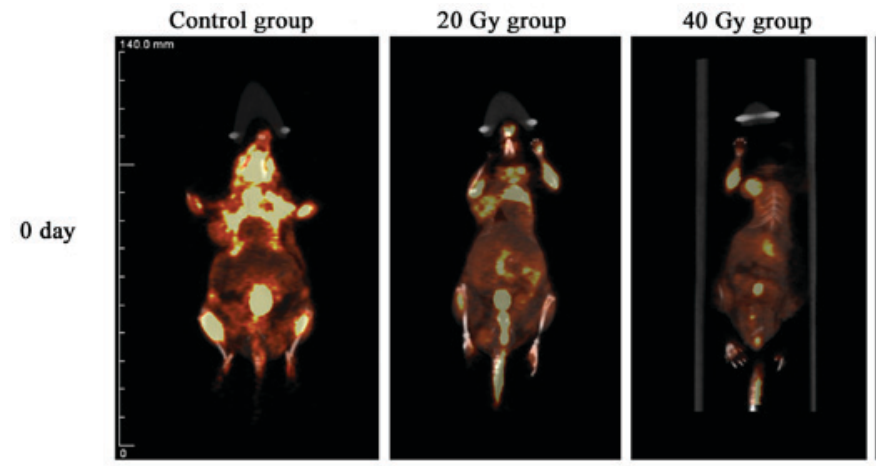

60 Gy group
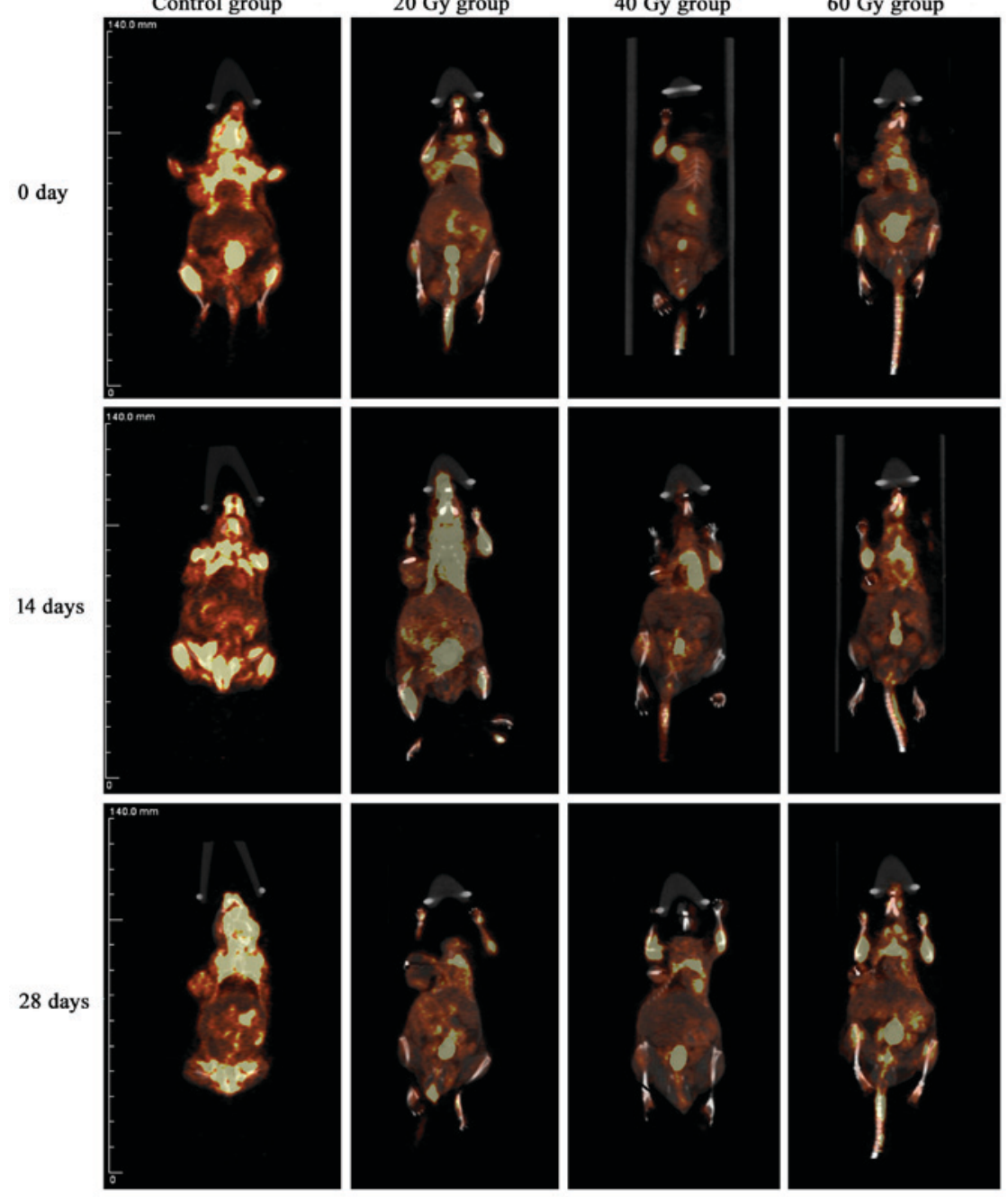

Figure 2. ${ }^{18} \mathrm{~F}$-FDG micro-PET/CT imaging in A549 xenografts following the administration of ${ }^{125} \mathrm{I}$-IBT. The ${ }^{18} \mathrm{~F}-\mathrm{FDG}$ uptake generally decreased over time within treatment group tumors, compared with the control group. ${ }^{18} \mathrm{~F}-\mathrm{FDG},{ }^{18} \mathrm{~F}$-fluorodeoxyglucose; micro-PET/CT, micro-positron emission tomography/computed tomography; ${ }^{125} \mathrm{I}$-IBT, iodine-125 interstitial brachytherapy.

${ }^{125}$ I-IBT suppresses mTOR, $c$-Myc, HIF-1 $\alpha$ and GLUT1 expression in A549 xenografts. The IHC staining patterns of mTOR, c-Myc, HIF-1 $\alpha$ and GLUT1 in A549 xenografts following ${ }^{125}$ I-IBT were presented in Fig. 4. In tumor cells, mTOR, c-Myc and HIF-1 $\alpha$ were expressed in the nucleus and cytoplasm, whereas GLUT1 was predominantly expressed in the cytomembrane and cytoplasm. Overall, the expression of mTOR, c-Myc, HIF-1 $\alpha$ and GLUT1 in xenografts was gradually downregulated as the radiation dose increased in the ${ }^{125}$ I-IBT treatment groups, compared with the controls (Fig. 5). The MOD of mTOR staining in the 60 Gy-group $(0.0092 \pm 0.0062)$ was markedly decreased compared with the other groups (control, $0.0198 \pm 0.0053 ; 20 \mathrm{~Gy}$, $0.0191 \pm 0.0096$ and $40 \mathrm{~Gy}, 0.0194 \pm 0.0062 ; \mathrm{P}<0.05$ ). In addition, the MOD of c-Myc staining was significantly lower in the 40 $(0.0025 \pm 0.0010)$ and $60 \mathrm{~Gy}(0.0006 \pm 0.0006)$ treatment groups, compared with the other groups (control, $0.0077 \pm 0.0020$ and $20 \mathrm{~Gy}, 0.0055 \pm 0.0029$; $\mathrm{P}<0.01)$. Furthermore, the MOD of HIF-1 $\alpha$ staining in the $40(0.0024 \pm 0.0021)$ and $60 \mathrm{~Gy}$ $(0.0018 \pm 0.0006)$ treatment groups was significantly than that of the control group $(0.0070 \pm 0.0058 ; \mathrm{P}<0.05)$. Finally, the MOD of GLUT1 staining in the 60 Gy $(0.0024 \pm 0.0025)$ group

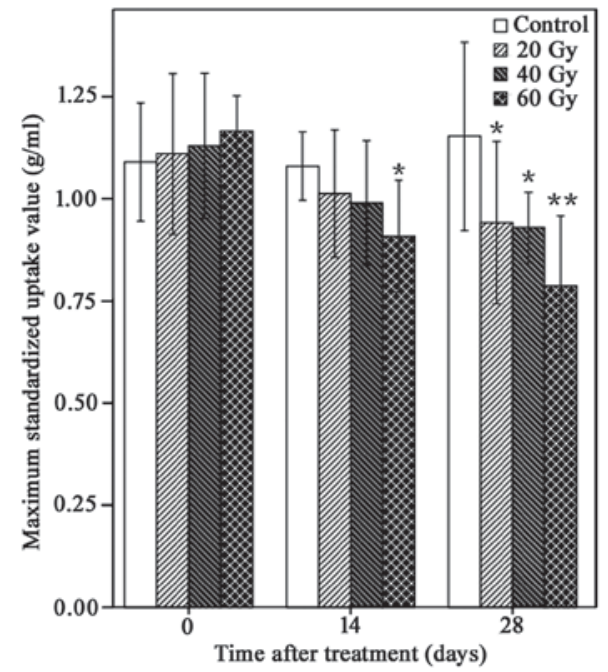

Figure 3. Comparison of ${ }^{18} \mathrm{~F}-\mathrm{FDG}$ uptake in A549 xenografts following the administration of ${ }^{125} \mathrm{I}$-IBT. The maximum standardized uptake value gradually reduced over time in the treatment groups compared with the control. Reduction was more marked as the radiation dose increased. ${ }^{*} \mathrm{P}<0.05$ ${ }^{* * *} \mathrm{P}<0.01$ vs. controls. ${ }^{18} \mathrm{~F}-\mathrm{FDG},{ }^{18} \mathrm{~F}$-fluorodeoxyglucose; ${ }^{125} \mathrm{I}$-IBT, iodine- 125 interstitial brachytherapy. 


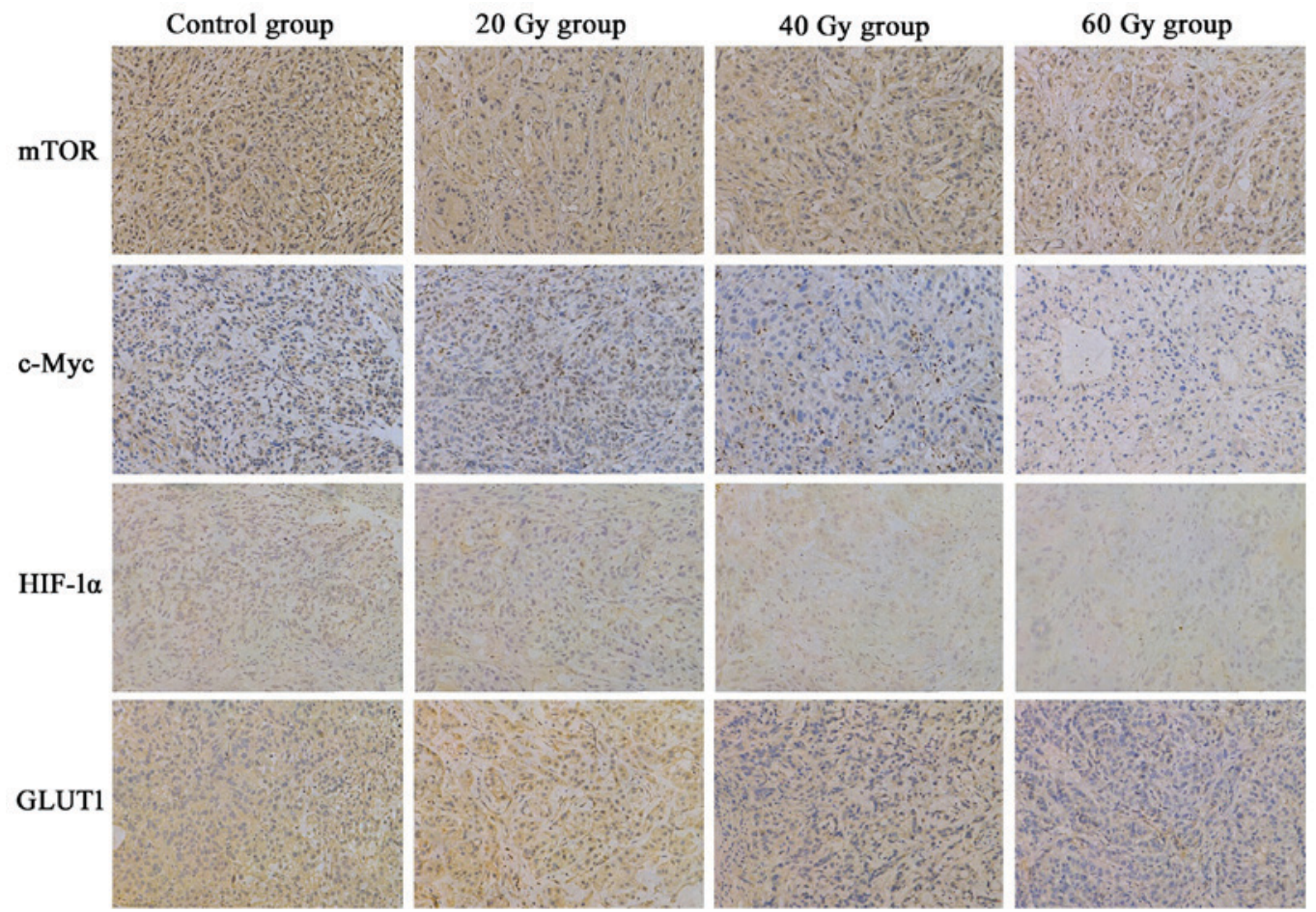

Figure 4. Immunohistochemical staining in A549 xenografts following ${ }^{125}$ I-IBT. mTOR, c-Myc, HIF-1 $\alpha$ and GLUT1 expression was downregulated in the treatment groups compared with the controls. Magnification, x200. mTOR, mammalian target of rapamycin; HIF-1 $\alpha$, hypoxia inducible factor-1 $\alpha$; GLUT1, glucose transporter $1 ;{ }^{125} \mathrm{I}-\mathrm{IBT}$, iodine-125 interstitial brachytherapy.

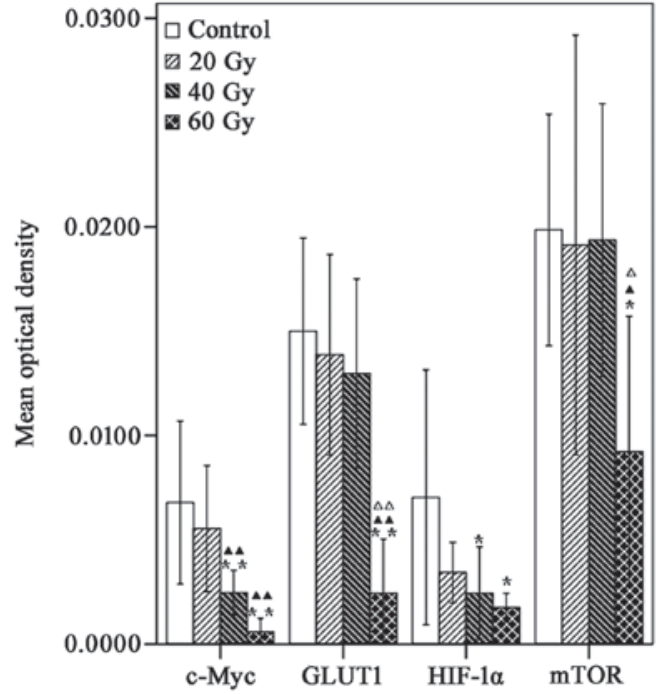

Figure 5. Comparison of mTOR, c-Myc, HIF-1 $\alpha$ and GLUT1 staining in A549 xenografts following ${ }^{125} \mathrm{I}-\mathrm{IBT}$. The mean optical densities of mTOR, c-Myc, HIF-1 $\alpha$ and GLUT1 gradually decreased as the radiation dose increased in treatment groups, compared with the controls. ${ }^{*} \mathrm{P}<0.05,{ }^{* *} \mathrm{P}<0.01$ vs. controls; ${ }^{\Delta} \mathrm{P}<0.05,{ }^{\Delta \boldsymbol{\Delta}} \mathrm{P}<0.01$ vs. 20 Gy group; ${ }^{\Delta} \mathrm{P}<0.05,{ }^{\Delta \Delta} \mathrm{P}<0.01$ vs. 40 Gy group. mTOR, mammalian target of rapamycin; HIF-1 $\alpha$, hypoxia inducible factor-1 $\alpha$ GLUT1, glucose transporter 1; ${ }^{125}$ I-IBT, iodine-125 interstitial brachytherapy.

was significantly decreased compared with the other (control,

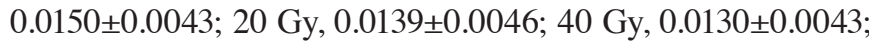
$\mathrm{P}<0.01)$. However, there were no significant differences between 20 Gy group and the control for mTOR, c-Myc, HIF-1 $\alpha$ or GLUT1 expression in A549 xenografts $(\mathrm{P}>0.05)$. Additionally, the $\mathrm{ESR}_{\mathrm{mTOR}}$, in the 20,40 and 60 Gy groups was 4.0, 2.5 and $53.8 \%$, respectively; the $\mathrm{ESR}_{\mathrm{c}-\mathrm{Myc}}$ was $28.6,67.5$ and $92.2 \%$, respectively; the $\mathrm{ESR}_{\mathrm{HIF-1a}}$ was $51.4,65.7$ and $74.3 \%$, respectively; and the $\mathrm{ESR}_{\mathrm{GLUT1}}$ was $7.3,13.3$ and $84.0 \%$, respectively. These data suggested that ${ }^{125}$ I-IBT may have suppressed the expression of mTOR, c-Myc, HIF-1 $\alpha$ and GLUT1 in A549 xenografts, and the suppression effect was increased as the radiation dose increased.

Relationships among tumor growth, ${ }^{18} \mathrm{~F}-\mathrm{FDG}$ uptake and expression of mTOR, $c-M y c, H I F-1 \alpha$ and GLUT1. The correlations among tumor growth, ${ }^{18} \mathrm{~F}-\mathrm{FDG}$ uptake and expression of mTOR, c-Myc, HIF-1 $\alpha$ and GLUT1 were presented in Fig. 6. There was a positive correlation between $\mathrm{TV}_{14}$ and SUVmax $14(\mathrm{r}=0.711), \mathrm{TV}_{28}$ and $\mathrm{SUVmax}_{14}(\mathrm{r}=0.682)$ and $\mathrm{TV}_{28}$ and $\mathrm{SUVmax}_{28}(\mathrm{r}=0.586)$, respectively $(\mathrm{P}<0.01)$. In addition, SUVmax 28 positively correlated to the MOD of c-Myc $(r=0.621)$ and GLUT1 $(r=0.546)$, respectively $(\mathrm{P}<0.01)$. However, the MOD of mTOR ( $r=0.243)$ and HIF-1 $\alpha(r=0.029)$ had no significant correlation with SUVmax ${ }_{28}(\mathrm{P}>0.05)$. Furthermore, the MOD of mTOR, c-Myc and GLUT1 had a significant correlation with each other $(\mathrm{P}<0.05$; Table I).

\section{Discussion}

In the present study, it was postulated that ${ }^{125}$ I-IBT may have suppressed NSCLC tumor growth by inhibiting the Warburg effect. Tumor growth and ${ }^{18} \mathrm{~F}-\mathrm{FDG}$ uptake was monitored in NSCLC A549 xenografts following ${ }^{125}$ I-IBT with a radiation dose of 20, 40 or $60 \mathrm{~Gy}$, and the relationships among them were also evaluated. It was demonstrated that both the TV and SUVmax of all treatment groups were decreased compared with controls following ${ }^{125}$ I-IBT, and these reductions were more marked as the time and radiation dose increased, which 
Table I. Associations among expression of mTOR, c-Myc, HIF-1 $\alpha$ and GLUT1 in A549 xenograft after ${ }^{125}{ }^{\text {I-IBT. }}$

\begin{tabular}{|c|c|c|c|}
\hline Protein name & $\begin{array}{l}\text { mTOR, correlation } \\
\text { coefficient (P-value) }\end{array}$ & $\begin{array}{l}\text { c-Myc, correlation } \\
\text { coefficient (P-value) }\end{array}$ & $\begin{array}{l}\text { HIF-1 } \alpha, \text { correlation } \\
\text { coefficient (P-value) }\end{array}$ \\
\hline
\end{tabular}

\begin{tabular}{|c|c|c|c|}
\hline c-Myc & $0.500^{\mathrm{a}}(0.013)$ & & \\
\hline HIF- $1 \alpha$ & $-0.003(0.988)$ & $0.365(0.079)$ & \\
\hline GLUT1 & $0.526^{\mathrm{b}}(0.008)$ & $0.614^{\mathrm{b}}(0.001)$ & $0.264(0.212)$ \\
\hline
\end{tabular}

${ }^{\mathrm{a}} \mathrm{P}<0.05,{ }^{\mathrm{b}} \mathrm{P}<0.01$. mTOR, mammalian target of rapamycin; HIF-1 $\alpha$, hypoxia inducible factor-1 $\alpha$; GLUT1, glucose transporter 1 ; ${ }^{125} \mathrm{I}-\mathrm{IBT}$, iodine-125 interstitial brachytherapy.
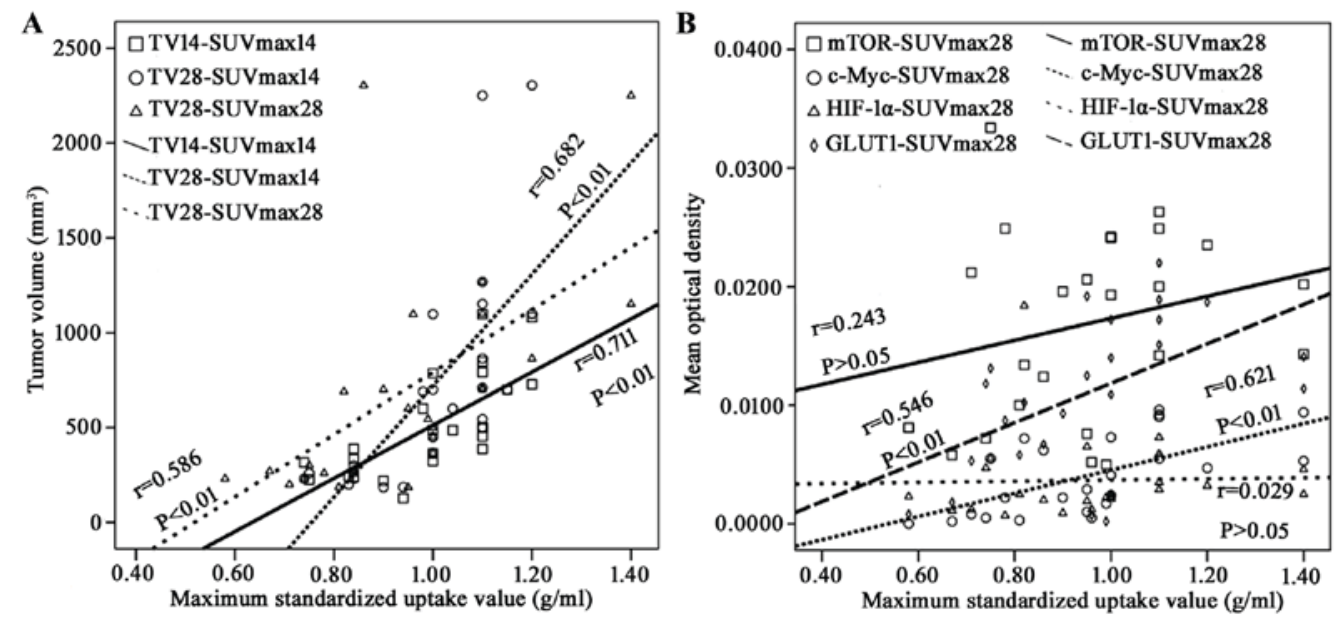

Figure 6. Relationships among tumor growth, ${ }^{18} \mathrm{~F}$-FDG uptake and expression of mTOR, c-Myc, HIF-1 $\alpha$ and GLUT1 in A549 xenografts following ${ }^{125} \mathrm{I}-\mathrm{IBT}$. (A) TV positively correlated to SUVmax on day $14(\mathrm{r}=0.711 ; \mathrm{P}<0.01)$ and day $28(\mathrm{r}=0.586 ; \mathrm{P}<0.01)$. TV on day 28 also correlated to SUVmax on day 14 $(\mathrm{r}=0.682$; $\mathrm{P}<0.01)$. (B) SUVmax positively correlated with the mean optical densities of $\mathrm{c}-\mathrm{Myc}(\mathrm{r}=0.621)$ and GLUT1 ( $\mathrm{r}=0.546)$ on day 28 , respectively $(\mathrm{P}<0.01)$. TV, tumor volume; SUVmax, maximum standardized uptake value; mTOR, mammalian target of rapamycin; HIF-1 $\alpha$, hypoxia inducible factor-1 $\alpha$; GLUT1, glucose transporter 1; ${ }^{125}$ I-IBT, iodine-125 interstitial brachytherapy.

was consistent with results obtained in previous studies of pancreatic carcinoma xenografts $(20,21)$. In addition, the TIR and FUAR increased more or less as the radiation dose increased, and TV positively correlated to SUVmax on day 14 and 28. On the basis of these results, it can be inferred that ${ }^{125}$ I-IBT may have reduced tumor growth by inhibiting the Warburg effect in NSCLC, and this inhibition increased in a dose-dependent manner more or less. Notably, it was revealed that SUVmax ${ }_{14}$ was positively correlated with $\mathrm{TV}_{28}(\mathrm{P}<0.05)$. This suggested that interim ${ }^{18} \mathrm{~F}-\mathrm{FDG} \mathrm{PET} / \mathrm{CT}$ may be a promising strategy to predict the anti-tumor efficacy of ${ }^{125} \mathrm{I}$-IBT in NSCLC in the early stages. ${ }^{18} \mathrm{~F}-\mathrm{FDG}$ PET/CT is currently used to predict chemotherapy and/or radiotherapy efficacy in lung cancer (16), as well as other malignancies $(22,23)$.

The Warburg effect is considered to involve multiple intricate mechanisms, including tumor microenvironment support, HIF stabilization, oncogene activation, tumor suppressor gene loss, cancer cell mitochondrial dysfunction, nuclear DNA mutations, epigenetic modifications, microRNA regulation, glutamine metabolism and post-translational modifications (24). HIF-1, a heterodimeric transcription factor consisting of $\alpha$ and $\beta$ subunits, is known to contribute to the adaptability of tumor cells to hypoxic conditions. It may increase the efficacy of the glycolytic pathway by upregulating the expression of several GLUTs and several other genes linked to aerobic glycolysis (25). For example, GLUT1 is ubiquitously expressed, but overexpressed in tumor cells and therefore increases the uptake of glucose. HIF regulation is predominantly dependent on the HIF- $1 \alpha$ subunit, that has a short half-life under normoxic conditions due to proteasomal degradation $(24,26,27)$. The phosphoinositide 3-kinase (PI3K)/protein kinase $\mathrm{B}(\mathrm{AKT})$ and the Ras/Raf/mitogen-activated protein kinase kinase (MAPKK)/extracellular regulated protein kinase (ERK) pathways are the major signaling pathways downstream from growth factor-bound receptor tyrosine kinases, which are linked to both growth control and glucose metabolism in tumor cells $(28,29)$. mTOR, a serine/threonine kinase, is an important downstream effector of AKT that stimulates the synthesis of HIF-1 $\alpha$ under normoxic conditions, further enhancing the expression of GLUT1 and glycolytic enzymes, including lactate dehydrogenase B and pyruvate kinase M2 (PKM2) $(10,24,30)$. The suppression of mTOR expression reduces glucose uptake in tumor cells (31). Furthermore, activated ERK induces the expression of MYC oncogene, which encodes a transcription factor c-Myc that is known to be aberrantly overexpressed in more than half of all human cancers (32). Similar to HIF-1, c-Myc activates GLUT1 and several glycolytic genes, resulting in increased glucose influx and higher glycolytic rates and contributes to the Warburg effect (33). In addition, activated c-Myc may work 
together with HIF-1 in glycolysis and OXPHOS regulation. Both HIF and c-Myc activate hexokinase 2 (HK2) and PDK1, leading to increased glycolytic rates and conversion of glucose to lactate (27).

In the present study, alterations in mTOR, c-Myc, HIF-1 $\alpha$ and GLUT1 expression was analyzed by IHC techniques, and the relationship among them was determined. The findings revealed that the xenograft expression of mTOR, c-Myc, HIF-1 $\alpha$ and GLUT1 decreased as the radiation dose increased, with the largest decrease observed in the $60 \mathrm{~Gy}$ group, compared with the controls. Furthermore, the $\mathrm{ESR}_{\mathrm{mTOR}}$, $\mathrm{ESR}_{\mathrm{c}-\mathrm{Myc}}, \mathrm{ESR}_{\mathrm{HIF}-1 \alpha}$ and $\mathrm{ESR}_{\mathrm{GLUT1}}$ was dose-dependently increased, and the MOD of mTOR, c-Myc and GLUT1 were significantly correlated with each other. These findings indicated that the Warburg effect was inhibited by ${ }^{125} \mathrm{I}-\mathrm{IBT}$, which may have resulted from mTOR, c-Myc, HIF-1 $\alpha$ and GLUT1 downregulation in NSCLC A549 xenografts.

Although the downregulation of the above molecules by ${ }^{125}$ I-IBT has not been explicitly defined in the present study, possible involvement of AKT and ERK-mediated signaling pathways may be suggested in accordance with a previous report demonstrating that ${ }^{125} \mathrm{I}$-IBT may downregulate epidermal growth factor receptor, inhibiting AKT activation in colorectal tumor cells (34). ${ }^{125}$ I-IBT has also been demonstrated to suppress vascular endothelial growth factor-A expression, and consequently inhibit ERK activation in nasopharyngeal tumor cells (35). It is plausible that AKT and ERK inactivation may have reduced the expression of $\mathrm{mTOR}$ and $\mathrm{c}-\mathrm{Myc}$, resulting in the downregulation of HIF-1 $\alpha$ and GLUT1 expression in the present study. In addition, Cron et al (36) reported that ${ }^{125}$ I seeds may significantly increase oxygen partial pressure within $24 \mathrm{~h}$ and even on day 3 . Furthermore, it may elevate blood perfusion from day 3 , in $2-4 \mathrm{~mm}$ of the surrounding liver tumor region (36). As a result, the improvement of the tumor hypoxic microenvironment by ${ }^{125}$ I-IBT may have also partially reduced the expression and/or accelerated the degradation of HIF-1 $\alpha$. However, Hu et al (37) demonstrated that ${ }^{125} \mathrm{I}$-IBT increases mitochondrial reactive oxygen species (ROS) production and consequently upregulates the expression of HIF-1 $\alpha$ in human colorectal cancer cells (37). Therefore, the downregulation of HIF-1 $\alpha$ by ${ }^{125}$ I-IBT may have been a synthetic action of above factors in NSCLC.

In addition, the correlation analysis between glucose metabolism and the expression of mTOR, c-Myc, HIF-1 $\alpha$ and GLUT1 in the present study revealed that the SUVmax 28 positively correlated with the MOD of c-Myc $(r=0.621)$ and GLUT1 ( $\mathrm{r}=0.546)$. This suggested that ${ }^{18} \mathrm{~F}-\mathrm{FDG}$ uptake may depend upon the overexpression of c-Myc and GLUT1 in xenografts, particularly c-Myc. GLUT1 is the major glucose transporter expressed in NSCLC. Although its overexpression is considered to be linked to ${ }^{18} \mathrm{~F}$-FDG uptake within tumors, it is unlikely that this is the sole or rate-limiting step in this process (38). The role of GLUT1 remains controversial in NSCLC; certain studies have reported a significant correlation $(39,40)$, whereas others have detected no correlation $(41,42)$. This may be attributed to the fact that ${ }^{18} \mathrm{~F}-\mathrm{FDG}$ uptake involves tumor blood flow and tracer delivery rate, in addition to expression of GLUT1. Once ${ }^{18} \mathrm{~F}-\mathrm{FDG}$ enters tumor cells, hexokinase and glucose-6-phosphatase (G6Pase) activity determines how much phosphorylated ${ }^{18} \mathrm{~F}-\mathrm{FDG}$ is trapped in cells (38). c-Myc, as a central regulator of cell growth and proliferation (43), targets genes involved in glucose transport, glycolysis, glutaminolysis and fatty acid synthesis (44). c-Myc activates GLUT1, as well as HK2 (27) and G6Pase (45), leading to increased uptake of ${ }^{18} \mathrm{~F}-\mathrm{FDG}$. Downregulation of c-Myc and GLUT1 suppresses the glycolytic pathway and glycolytic enzyme activity, leading to ${ }^{18} \mathrm{~F}-\mathrm{FDG}$ uptake inhibition (46).

However, it was noted that in the present study, no statistical differences between TV, SUVmax ${ }_{14}$ and SUVmax 28 were observed among the treatment groups, or between the TV and the MOD of mTOR, c-Myc, HIF-1 $\alpha$ and GLUT1 in the 20 Gy and control group. This may have been due to the short investigation duration of 28 days, as ${ }^{125}$ I-IBT is typically delivered over $>180$ days (21). Additionally, $60 \mathrm{~Gy}$ is a relatively low radiation dose, considering that the clinically effective dose is typically 100-160 Gy (3-6). Furthermore, there were no significant correlations between HIF-1 $\alpha$ expression and the other detected proteins, or between the SUVmax 28 and the MOD of mTOR and HIF-1 $\alpha$ $(\mathrm{P}>0.05)$. This may be attributed in part to the complex, multifactorial regulation of $\mathrm{HIF}-1 \alpha$ as detailed above. In addition, it suggested that the AKT/mTOR pathway may have less influence on the Warburg effect in NSCLC, compared with the ERK/c-Myc pathway.

Certain limitations of the present study should be considered. First, other key molecules associated with glycolysis were not examined, such as PKM2 and HK2. Second, the expression of glycolysis-associated molecules was only evaluated using IHC rather than via western blotting and/or reverse transcription-quantitative polymerase chain reaction assays for transcriptional and translational expression analyses, respectively. Third, A549 cells harbor the mutant $\mathrm{K}$-ras gene, and the Ras/Raf/ERK signaling pathway is critical for tumor proliferation. Therefore, multiple NSCLC cells, including those with the wild-type K-ras gene, should be used to confirm the results of the present study.

In conclusion, the present study suggested that ${ }^{125} \mathrm{I}$-IBT may have reduced tumor growth via Warburg effect inhibition, and this inhibitory effect increased as the radiation dose increased. Warburg effect inhibition may have resulted from mTOR, c-Myc, HIF-1 $\alpha$ and GLUT1 expression downregulation, particularly c-Myc and GLUT1, in NSCLC A549 xenografts. Further research is required to clearly delineate the exact mechanisms underlying Warburg effect inhibition by ${ }^{125}$ I-IBT in NSCLC.

\section{Acknowledgements}

The authors would like to thank Mr. Jiong Yu for the cell culture assistance, Mr. Li Jiang for helping establishing the animal model and Mr. Hui Chen for assistance in animal imaging.

\section{Funding}

The present study was supported by the Zhejiang Provincial Natural Science Foundation (grant no. LY15H180007) and Zhejiang Province Medical Health Science Foundation of China (grant nos. 2015KYB153, 2016KYB099 and 2017KY061). 


\section{Availability of data and materials}

The datasets used and/or analyzed during the current study are available from the corresponding author on reasonable request.

\section{Authors' contributions}

JZ established animal models, performed animal imaging and drafted the manuscript. YZ was responsible for cell culture and participated in preparation of animal model and imaging. MD, JY and WW participated in the iodine-125 seed implantation and immunohistochemistry. LT conceived and designed the study, performed the statistical analysis and revised the manuscript. All authors read and approved the final manuscript.

\section{Ethics approval and consent to participate}

The animals used in the present study received humane care in compliance with the Guideline to the Care and Use of Experimental Animals established by the Medical Ethical Committee on animal experiments of the Zhejiang University.

\section{Patient consent for publication}

Not applicable.

\section{Competing interests}

The authors declare that they have no competing interests.

\section{References}

1. Torre LA, Bray F, Siegel RL, Ferlay J, Lortet-Tieulent J and Jemal A: Global cancer statistics, 2012. CA Cancer J Clin 65: 87-108, 2015.

2. Miller KD, Siegel RL, Lin CC, Mariotto AB, Kramer JL, Rowland JH, Stein KD, Alteri R and Jemal A: Cancer treatment and survivorship statistics, 2016. CA Cancer J Clin 66: 271-289, 2016.

3. Huo X, Wang H, Yang J, Li X, Yan W, Huo B, Zheng G, Chai S, Wang J, Guan Z and Yu Z: Effectiveness and safety of CT-guided (125)I seed brachytherapy for postoperative locoregional recurrence in patients with non-small cell lung cancer. Brachytherapy 15: 370-380, 2016.

4. Li W, Guan J, Yang L, Zheng X, Yu Y and Jiang J: Iodine-125 brachytherapy improved overall survival of patients with inoperable stage III/IV non-small cell lung cancer versus the conventional radiotherapy. Med Oncol 32: 395, 2015.

5. Yu X, Li J, Zhong X and He J: Combination of Iodine-125 brachytherapy and chemotherapy for locally recurrent stage III non-small cell lung cancer after concurrent chemoradiotherapy. BMC Cancer 15: 656, 2015.

6. Li W, Dan G, Jiang J, Zheng Y, Zheng X and Deng D: Repeated iodine-125 seed implantations combined with external beam radiotherapy for the treatment of locally recurrent or metastatic stage III/IV non-small cell lung cancer: A retrospective study. Radiat Oncol 11: 119, 2016.

7. Chen HH, Jia RF, Yu L, Zhao MJ, Shao CL and Cheng WY: Bystander effects induced by continuous low-dose-rate 125I seeds potentiate the killing action of irradiation on human lung cancer cells in vitro. Int J Radiat Oncol Biol Phys 72: 1560-1566, 2008.

8. Qu A, Wang H, Li J, Wang J, Liu J, Hou Y, Huang L and Zhao Y: Biological effects of (125)i seeds radiation on A549 lung cancer cells: G2/M arrest and enhanced cell death. Cancer Invest 32 : 209-217, 2014

9. Wang Z, Zhao Z, Lu J, Chen Z, Mao A, Teng G and Liu F: A comparison of the biological effects of $125 \mathrm{I}$ seeds continuous low-dose-rate radiation and 60Co high-dose-rate gamma radiation on non-small cell lung cancer cells. PLoS One 10: e133728, 2015.
10. Vander Heiden MG, Cantley LC and Thompson CB: Understanding the Warburg effect: The metabolic requirements of cell proliferation. Science 324: 1029-1033, 2009.

11. Ganapathy V, Thangaraju M and Prasad PD: Nutrient transporters in cancer: Relevance to Warburg hypothesis and beyond. Pharmacol Ther 121: 29-40, 2009.

12. Liu J, Dong M, Sun X, Li W, Xing L and Yu J: Prognostic value of 18F-FDG PET/CT in surgical non-small cell lung cancer: A meta-analysis. PLoS One 11: e146195, 2016.

13. Madsen PH, Holdgaard PC, Christensen JB and Høilund-Carlsen PF: Clinical utility of F-18 FDG PET-CT in the initial evaluation of lung cancer. Eur J Nucl Med Mol Imaging 43: 2084-2097, 2016.

14. Ruilong Z, Daohai X, Li G, Xiaohong W, Chunjie W and Lei T: Diagnostic value of 18F-FDG-PET/CT for the evaluation of solitary pulmonary nodules: A systematic review and meta-analysis. Nucl Med Commun 38: 67-75, 2017.

15. Sheikhbahaei S, Mena E, Yanamadala A, Reddy S, Solnes LB, Wachsmann J and Subramaniam RM: The value of FDG PET/CT in treatment response assessment, follow-up, and surveillance of lung cancer. AJR Am J Roentgenol 208: 420-433, 2017.

16. Cremonesi M, Gilardi L, Ferrari ME, Piperno G, Travaini LL, Timmerman R, Botta F, Baroni G, Grana CM, Ronchi S, et al: Role of interim ${ }^{18} \mathrm{~F}$-FDG-PET/CT for the early prediction of clinical outcomes of non-small cell lung cancer (NSCLC) during radiotherapy or chemo-radiotherapy. A systematic review. Eur J Nucl Med Mol Imaging 44: 1915-1927, 2017.

17. Allen KT, Chin-Sinex H, DeLuca T, Pomerening JR, Sherer J, Watkins JR, Foley J, Jesseph JM and Mendonca MS: Dichloroacetate alters Warburg metabolism, inhibits cell growth, and increases the X-ray sensitivity of human A549 and H1299 NSC lung cancer cells. Free Radic Biol Med 89: 263-273, 2015.

18. Lv XB, Liu L, Cheng C, Yu B, Xiong L, Hu K, Tang J, Zeng L and Sang Y: SUN2 exerts tumor suppressor functions by suppressing the Warburg effect in lung cancer. Sci Rep 5: 17940, 2015.

19. Liu T and Yin H: PDK1 promotes tumor cell proliferation and migration by enhancing the Warburg effect in non-small cell lung cancer. Oncol Rep 37: 193-200, 2017.

20. Ma JX, Jin ZD, Si PR, Liu Y, Lu Z, Wu HY, Pan X, Wang LW, Gong YF, Gao J and Zhao-shen L: Continuous and low-energy 125I seed irradiation changes DNA methyltransferases expression patterns and inhibits pancreatic cancer tumor growth. J Exp Clin Cancer Res 30: 35, 2011.

21. Jian L, Zhongmin W, Kemin C, Yunfeng $Z$ and Gang H: MicroPET-CT evaluation of interstitial brachytherapy in pancreatic carcinoma xenografts. Acta Radiol 54: 800-804, 2013.

22. Subocz E, Hałka J and Dziuk M: The role of FDG-PET in Hodgkin lymphoma. Contemp Oncol (Pozn) 21: 104-114, 2017.

23. Cremonesi M, Garibaldi C, Timmerman R, Ferrari M, Ronchi S, Grana CM, Travaini L, Gilardi L, Starzynska A, Ciardo D, et al: Interim ${ }^{18} \mathrm{~F}$-FDG-PET/CT during chemo-radiotherapy in the management of oesophageal cancer patients. A systematic review. Radiother Oncol 125: 200-212, 2017.

24. Upadhyay M, Samal J, Kandpal M, Singh OV and Vivekanandan P: The Warburg effect: Insights from the past decade. Pharmacol Ther 137: 318-330, 2013.

25. Thorens B and Mueckler M: Glucose transporters in the 21st Century. Am J Physiol Endocrinol Metab 298: E141-E145, 2010.

26. Lu H, Forbes RA and Verma A: Hypoxia-inducible factor 1 activation by aerobic glycolysis implicates the Warburg effect in carcinogenesis. J Biol Chem 277: 23111-23115, 2002.

27. Chen X, Qian Y and Wu S: The Warburg effect: Evolving interpretations of an established concept. Free Radic Biol Med 79: 253-263, 2015.

28. Dang CV: Links between metabolism and cancer. Genes Dev 26: 877-890, 2012.

29. Hsieh AL, Walton ZE, Altman BJ, Stine ZE and Dang CV: MYC and metabolism on the path to cancer. Semin Cell Dev Biol 43: $11-21,2015$

30. Makinoshima H, Takita M, Saruwatari K, Umemura S, Obata Y, Ishii G, Matsumoto S, Sugiyama E, Ochiai A, Abe R, et al: Signaling through the phosphatidylinositol 3-kinase $(\mathrm{PI} 3 \mathrm{~K}) / \mathrm{mammalian}$ target of rapamycin (mTOR) axis is responsible for aerobic glycolysis mediated by glucose transporter in epidermal growth factor receptor (EGFR)-mutated lung adenocarcinoma. J Biol Chem 290: 17495-17504, 2015.

31. Toschi A, Lee E, Thompson S, Gadir N, Yellen P, Drain CM, Ohh M and Foster DA: Phospholipase D-mTOR requirement for the Warburg effect in human cancer cells. Cancer Lett 299: 72-79, 2010. 32. Dang CV: MYC on the path to cancer. Cell 149: 22-35, 2012. 
33. Dang CV, Kim JW, Gao P and Yustein J: The interplay between MYC and HIF in cancer. Nat Rev Cancer 8: 51-56, 2008.

34. Liu J, Wang H, Qu A, Li J, Zhao Y and Wang J: Combined effects of $\mathrm{C} 225$ and 125-iodine seed radiation on colorectal cancer cells. Radiat Oncol 8: 219, 2013.

35. Tian Y, Xie Q, Tian Y, Liu Y, Huang Z, Fan C, Hou B, Sun D, Yao K and Chen T: Radioactive ${ }^{125}$ I seed inhibits the cell growth, migration, and invasion of nasopharyngeal carcinoma by triggering DNA damage and inactivating VEGF-A/ERK signaling. PLoS One 8: e74038, 2013.

36. Cron GO, Beghein N, Crokart N, Chavée E, Bernard S, Vynckier S, Scalliet $\mathrm{P}$ and Gallez B: Changes in the tumor microenvironment during low-dose-rate permanent seed implantation iodine-125 brachytherapy. Int J Radiat Oncol Biol Phys 63: 1245-1251, 2005.

37. Hu L, Wang $\mathrm{H}$, Huang $\mathrm{L}$, Zhao $\mathrm{Y}$ and Wang J: The protective roles of ROS-mediated mitophagy on ${ }^{125} \mathrm{I}$ seeds radiation induced cell death in HCT116 cells. Oxid Med Cell Longev 2016: 9460462, 2016.

38. Brown RS, Leung JY, Kison PV, Zasadny KR, Flint A and Wahl RL: Glucose transporters and FDG uptake in untreated primary human non-small cell lung cancer. J Nucl Med 40: $556-565,1999$.

39. van Baardwijk A, Dooms C, van Suylen RJ, Verbeken E, Hochstenbag M, Dehing-Oberije C, Rupa D, Pastorekova S, Stroobants S, Buell U, et al: The maximum uptake of (18) F-deoxyglucose on positron emission tomography scan correlates with survival, hypoxia inducible factor-1alpha and GLUT-1 in non-small cell lung cancer. Eur J Cancer 43: 1392-1398, 2007.

40. Suzawa N, Ito M, Qiao S, Uchida K, Takao M, Yamada T, Takeda K and Murashima S: Assessment of factors influencing FDG uptake in non-small cell lung cancer on PET/CT by investigating histological differences in expression of glucose transporters 1 and 3 and tumour size. Lung Cancer 72: 191-198, 2011.
41. Chung JK, Lee YJ, Kim SK, Jeong JM, Lee DS and Lee MC: Comparison of $[18 \mathrm{~F}]$ fluorodeoxyglucose uptake with glucose transporter-1 expression and proliferation rate in human glioma and non-small-cell lung cancer. Nucl Med Commun 25: 11-17, 2004.

42. de Geus-Oei LF, van Krieken JH, Aliredjo RP, Krabbe PF, Frielink C, Verhagen AF, Boerman OC and Oyen WJ: Biological correlates of FDG uptake in non-small cell lung cancer. Lung Cancer 55: 79-87, 2007.

43. Dang CV: Rethinking the Warburg effect with Myc micromanaging glutamine metabolism. Cancer Res 70: 859-862, 2010.

44. Morrish F, Isern N, Sadilek M, Jeffrey M and Hockenbery DM: c-Myc activates multiple metabolic networks to generate substrates for cell-cycle entry. Oncogene 28: 2485-2491, 2009.

45. Collier JJ, Doan TT, Daniels MC, Schurr JR, Kolls JK and Scott DK: c-Myc is required for the glucose-mediated induction of metabolic enzyme genes. J Biol Chem 278: 6588-6595, 2003.

46. Broecker-Preuss M, Becher-Boveleth N, Bockisch A, Dührsen U and Müller S: Regulation of glucose uptake in lymphoma cell lines by c-MYC- and PI3K-dependent signaling pathways and impact of glycolytic pathways on cell viability. J Transl Med 15: $158,2017$.

This work is licensed under a Creative Commons Attribution-NonCommercial-NoDerivatives 4.0 International (CC BY-NC-ND 4.0) License. 